\title{
Picosecond carrier recombination dynamics in chalcogen-hyperdoped silicon
}

\section{Citation}

Sher, Meng-Ju, Christie B. Simmons, Jacob J. Krich, Austin J. Akey, Mark T. Winkler, Daniel Recht, Tonio Buonassisi, Michael J. Aziz, and Aaron M. Lindenberg. 2014. "Picosecond Carrier Recombination Dynamics in Chalcogen-Hyperdoped Silicon." Appl. Phys. Lett. 105 (5) (August 4): 053905. Portico. doi:10.1063/1.4892357.

\section{Published Version}

doi:10.1063/1.4892357

\section{Permanent link}

http://nrs.harvard.edu/urn-3:HUL.InstRepos:22970215

\section{Terms of Use}

This article was downloaded from Harvard University's DASH repository, and is made available under the terms and conditions applicable to Other Posted Material, as set forth at http:// nrs.harvard.edu/urn-3:HUL.InstRepos:dash.current.terms-of-use\#LAA

\section{Share Your Story}

The Harvard community has made this article openly available.

Please share how this access benefits you. Submit a story.

\section{Accessibility}




\title{
Picosecond carrier recombination dynamics in chalcogen-hyperdoped silicon
}

\author{
Meng-Ju Sher ${ }^{1, \text { a) }}$ Christie B. Simmons, ${ }^{2}$ Jacob J. Krich, ${ }^{3}$ Austin J. Akey, ${ }^{2}$ Mark T. Winkler, ${ }^{2}$ \\ Daniel Recht, ${ }^{4}$ Tonio Buonassisi, ${ }^{2}$ Michael J. Aziz, ${ }^{4}$ and Aaron M. Lindenberg ${ }^{1,5,6}$ \\ ${ }^{1}$ Stanford Institute for Materials and Energy Sciences, SLAC National Accelerator Laboratory, Menlo Park, \\ California 94025, USA \\ ${ }^{2}$ Department of Mechanical Engineering, Massachusetts Institute of Technology, Cambridge, \\ Massachusetts 02139, USA \\ ${ }^{3}$ Department of Physics, University of Ottawa, Ottawa, Ontario KIN 6N5, Canada \\ ${ }^{4}$ Harvard School of Engineering and Applied Sciences, Cambridge, Massachusetts 02138, USA \\ ${ }^{5}$ PULSE Institute, SLAC National Accelerator Laboratory, Menlo Park, California 94025, USA \\ ${ }^{6}$ Department of Materials Science and Engineering, Stanford University, Stanford, California 94305, USA
}

(Received 20 June 2014; accepted 20 July 2014; published online 7 August 2014)

\begin{abstract}
Intermediate-band materials have the potential to be highly efficient solar cells and can be fabricated by incorporating ultrahigh concentrations of deep-level dopants. Direct measurements of the ultrafast carrier recombination processes under supersaturated dopant concentrations have not been previously conducted. Here, we use optical-pump/terahertz-probe measurements to study carrier recombination dynamics of chalcogen-hyperdoped silicon with sub-picosecond resolution. The recombination dynamics is described by two exponential decay time scales: a fast decay time scale ranges between 1 and 200 ps followed by a slow decay on the order of $1 \mathrm{~ns}$. In contrast to the prior theoretical predictions, we find that the carrier lifetime decreases with increasing dopant concentration up to and above the insulator-to-metal transition. Evaluating the material's figure of merit reveals an optimum doping concentration for maximizing performance. (C) 2014 AIP Publishing LLC.
\end{abstract}

[http://dx.doi.org/10.1063/1.4892357]

Incorporating a non-equilibrium concentration of deeplevel dopants creates materials with unique optical, electronic, and optoelectronic properties, with potential applications to infrared detectors and intermediate-band photovoltaic (IBPV) devices. ${ }^{1-3}$ Defect engineering enables control of the band structure of semiconductors, leading to an additional energy band within the band gap and possibilities for harvesting additional energy from low energy photons which normally would not be absorbed by a traditional single-junction solar cell. ${ }^{4}$ Many potential IB materials have been developed, since the IBPV concept was proposed in 1997, but none has shown efficiency improvements. ${ }^{5}$ In this work, we study carrier dynamics to understand the impact of ultrahigh dopant concentration $(N)$ on carrier lifetime, investigating a theoretically proposed hypothesis that nonradiative recombination is suppressed at dopant concentrations sufficient to induce an insulator-to-metal transition (IMT). ${ }^{6}$ We use optical-pump/ terahertz-probe techniques to directly measure carrier recombination time-scales in chalcogen-hyperdoped silicon (HD$\mathrm{Si}$ ) and show that lifetime recovery does not occur above the IMT. It is shown nevertheless that there is an optimal dopant concentration at which the IBPV performance figure of merit is optimized.

Determining carrier lifetime in an IB material is critical because only carriers with sufficiently long lifetime can be extracted from the material before the energy is lost to carrier recombination. Deep-level states exhibit strong ShockleyRead-Hall nonradiative recombination; the recombination rate increases with density of the deep-level states and hence the

a)msher@stanford.edu carrier lifetime is reduced. ${ }^{7}$ The lifetime recovery proposal hypothesizes that when carrier concentration is sufficiently high such that the deep-level states form a delocalized band nonradiative recombination is suppressed. ${ }^{6,89}$ Many research efforts have focused on fabricating materials with $N$ above the IMT for IB formation, but the lifetime recovery theory has not been carefully tested. From a more general perspective, knowledge of carrier dynamics in an IB material enables evaluation of the material's potential for a successful IBPV. A figure of merit proposed in Ref. 10 quantifies the tradeoff between the carrier lifetime and the sub-band-gap absorptivity, is a property of the IB material alone, and allows determination of the potential of an IB material to make a highly efficient IBPV.

Chalcogen-hyperdoped Si has been a proposed IB material and is ideal for this study since many relevant material properties have been previously reported. When heavy chalcogen $(\mathrm{S}, \mathrm{Se}$, or $\mathrm{Te}$ ) dopants are incorporated into $\mathrm{Si}$ at concentrations near atomic percent level, the resulting material exhibits strong sub-band-gap light absorption. ${ }^{11,12}$ The IMT has been experimentally observed in this system at $N$ around $3 \times 10^{20} \mathrm{~cm}^{-3} \cdot{ }^{13}$ Microwave photoconductivity decay and low-temperature photoconductivity measurements on carrier dynamics in this material lack the time-resolution required to measure the lifetime directly. ${ }^{14-16}$ Similar to microwaves, $\mathrm{THz}$ radiation is long-wavelength electromagnetic radiation and is sensitive to free charge carriers. Moreover, subpicosecond-duration $\mathrm{THz}$ pulses can be straightforwardly generated, enabling direct mapping of the carrier recombination dynamics on picosecond time scales. ${ }^{17}$

To fabricate S- and Se-hyperdoped Si samples, we use ion-implantation followed by nanosecond pulsed-laser melting 
and rapid resolidification, a well-established method for fabricating high quality single crystalline samples with $N$ above the equilibrium solid-solubility limit. ${ }^{18}$ Variation in $N$ is achieved by varying the implantation dose ranging from $1 \times 10^{14}$ to $1 \times 10^{16}$ ions $/ \mathrm{cm}^{2}$. The $\mathrm{Si}$ wafers used are $800-\mu \mathrm{m}$ thick, double-side polished and p-type (boron doped with resistivity of $10-30 \Omega \cdot \mathrm{cm}$ ). The surface of the wafers is amorphized by the implants, so following ion-implantation, we irradiate the samples with four $\mathrm{XeCl}$ excimer laser pulses $(308 \mathrm{~nm}$ wavelength, $25 \mathrm{~ns}$ in pulse duration, and $2 \mathrm{~mm} \times 2 \mathrm{~mm}$ spot size) to restore the crystallinity. Detailed fabrication parameters ${ }^{13}$ and crystalline characterization ${ }^{19}$ are documented elsewhere. The resulting hyperdoped material is approximately 200-nm thick and has $N$ ranging from $3.6 \times 10^{18}$ to $4.9 \times 10^{20}$ atoms $/ \mathrm{cm}^{3}$. 13,20,21

We perform time-resolved optical-pump/THz-probe measurements, monitoring $\mathrm{THz}$ transmission as a function of time up to $1 \mathrm{~ns}$ after pump excitation. Amplified femtosecond laser pulses (800-nm center wavelength, $1.7-\mathrm{mJ}$ pulse energy, $50-\mathrm{fs}$ pulse duration, and $1-\mathrm{kHz}$ repetition rate) are used for $\mathrm{THz}$ generation, detection and photoexcitation. The $\mathrm{THz}$ probe pulses are generated from a two-color laserinduced gas plasma and detected by electro-optic sampling in a $1.0-\mathrm{mm}$ thick $\langle 110\rangle \mathrm{ZnTe}$ crystal, directly measuring the transmitted electric field of the probe pulses. ${ }^{22}$ To selectively excite the thin hyperdoped layer and avoid carrier generation in the Si substrate, we frequency double the pump pulses to a center wavelength of $400 \mathrm{~nm}$ using a thin beta barium borate (BBO) crystal. Samples are excited from an oblique angle, focused to a 2-mm diameter spot with fluence of $20 \mu \mathrm{J} / \mathrm{cm}^{2}$. In order to selectively excite carriers in the IB, we also perform pump-probe experiments with sub-band gap excitation. Pump pulses at $2300 \mathrm{~nm}$ are generated from an optical parametric amplifier and focused on the samples at a fluence of $64 \mu \mathrm{J} / \mathrm{cm}^{2}$. After photoexcitation, no phase shift is observed in the transmitted $\mathrm{THz}$ pulse and we record transmission change at the maximum of the $\mathrm{THz}$ pulse (shown in supplementary material). ${ }^{23}$

We describe first how the time-dependent changes in $\mathrm{THz}$ transmission reflect changes in conductivity, $\Delta \sigma$, and in the density of free carriers, $\Delta n .^{24,25}$ The amplitude transmission $T$ of a thin film with conductivity $\sigma$ is given by $T(t)=\frac{2}{1+n_{\mathrm{Si}}+Z_{0} d \sigma(t)}$, where $d$ is the thickness of the hyperdoped layer, approximately $200 \mathrm{~nm}, n_{\mathrm{Si}}$ is the index of refraction of the Si substrate $\left(n_{\mathrm{Si}}=3.41\right),{ }^{26}$ and $Z_{0}$ is the impedance of free space $\left(Z_{0}=377 \Omega\right) .{ }^{24}$ The normalized change in $\mathrm{THz}$ transmission as a function of time is

$$
\frac{-\Delta T(t)}{T_{0}}=\frac{\sigma(t)-\sigma_{0}}{\sigma(t)+\frac{1+n_{\mathrm{Si}}}{Z_{0} d}},
$$

where $\sigma_{0}$ is the initial conductivity in the sample before photoexcitation and $T_{0}$ is the initial $\mathrm{THz}$ transmission. We assume that the mobility of carriers, $\mu$, is invariant on the time scale we are probing, ${ }^{27,28}$ so that the change in conductivity is directly proportional to the change in carrier concentration $\sigma(t)-\sigma_{0}=\Delta \sigma(t)=q \mu \Delta n(t)$, where $q$ is the elementary charge. Both free electrons and free holes attenuate $\mathrm{THz}$ transmission, but since the electron mobility is larger than the hole mobility, we assume the signal is dominated by the free electron density. We show later by only generating free electrons with 2300-nm excitation wavelength that indeed the free electrons are the dominant contribution to the detected $\mathrm{THz}$ conductivity signal. By solving Eq. (1) for $\Delta \sigma(t)$ and substituting with $q \mu \Delta n(t)$, we obtain the normalized change in carrier concentration as a function of $\mathrm{THz}$ transmission as follows:

$$
\frac{\Delta n(t)}{n_{0}+A}=\frac{-\Delta T(t) / T_{0}}{1+\Delta T(t) / T_{0}},
$$

where $A=\frac{1+n_{s i}}{Z_{0} d q \mu}$. Using Eq. (2), we calculate the change in carrier concentration as a function of time.

Figure 1 shows the measured time-dependent carrier concentration in S-hyperdoped Si after 400-nm photoexcitation. We also study the carrier dynamics in Se-hyperdoped $\mathrm{Si}$ obtaining similar results (see supplementary material). ${ }^{23} \mathrm{In}$ Figure 1, the fluence of the pump pulse is fixed at $20 \mu \mathrm{J} / \mathrm{cm}^{2}$, so $\Delta n$ is the same across all samples $\left(\Delta n \approx 2 \times 10^{18}\right.$ carriers/ $\mathrm{cm}^{3}$ per pulse), but we find that recombination dynamics is independent of pump fluence in the fluence range studied between 20 and $200 \mu \mathrm{J} / \mathrm{cm}^{2}$. With increasing $N$, carriers relax toward equilibrium faster. We model our data with a biexponential decay convoluted with the instrument's response, $G\left(t-t_{0}\right) \otimes\left(a_{1} \exp \left(-\left(t-t_{0}\right) / \tau_{\text {fast }}\right)+a_{2} \exp \left(\left(t-t_{0}\right) / \tau_{\text {slow }}\right)\right)$, where $a_{1}$ and $a_{2}$ show the relative weights $\left(a_{1}+a_{2}=1\right){ }^{29}$ The instrument's response $G\left(t-t_{0}\right)$ is determined by the geometric crossing angle of the pump and the probe beam and is a Gaussian function with 700 fs full-width-half-maximum centered at time zero $t_{0}$. Fit results are shown in the dashed lines in Figure 1. Figure 2 summarizes the dependence of lifetime on $N$. The best fit result suggests $\tau_{\text {slow }}$ is on the order of $1 \mathrm{~ns}$ and $\tau_{\text {fast }}$ decreases with increasing dopant concentration approaching $1 \mathrm{ps}$ at the highest concentrations measured. The best fit values for $a_{1}$ increase with $N$ and are given in supplementary Table $1 ;^{23}$ in the high $N$ limit, $\tau_{\text {slow }}$ plays a small role in the photoconductivity decay. Further discussion of the origin of $\tau_{\text {slow }}$ and $\tau_{\text {fast }}$ is presented below.

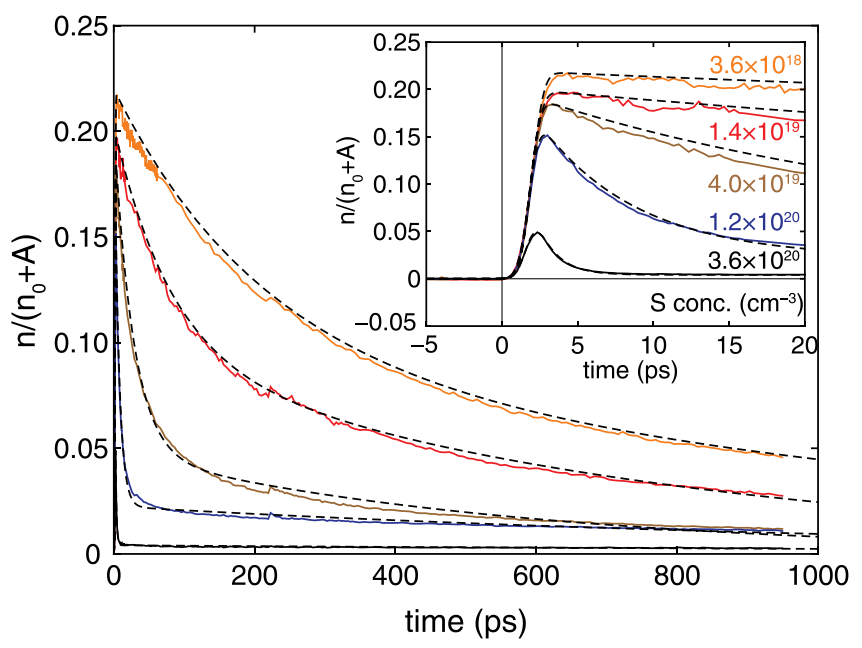

FIG. 1. Change in free carrier concentration in S-hyperdoped Si after photoexcitation with a fs-laser pulse at $400 \mathrm{~nm}$. Inset shows short time scale dynamics. We model the data with a bi-exponential decay and the dashed lines show the best fits. 


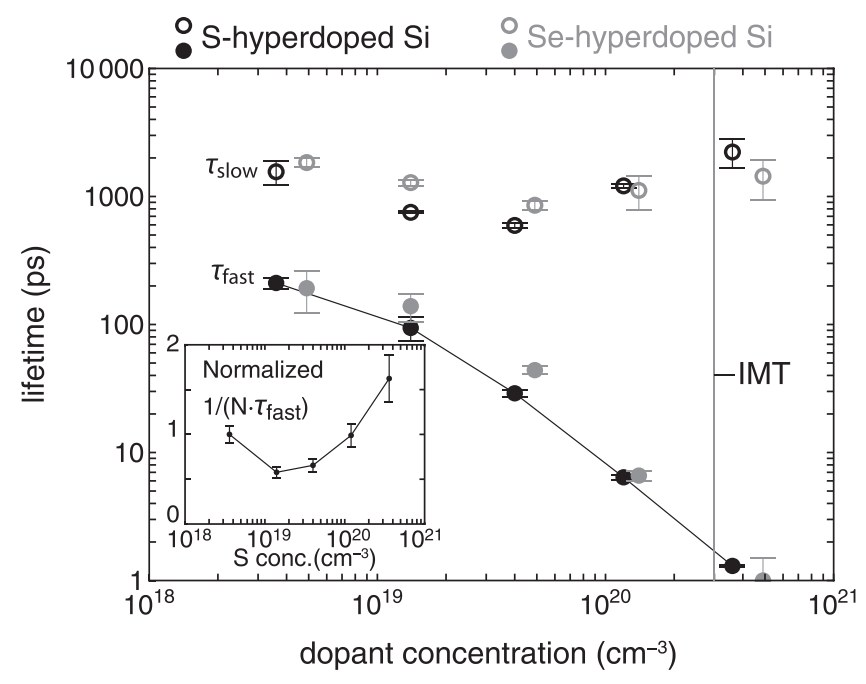

FIG. 2. Carrier lifetime as a function of dopant concentration for both Shyperdoped (black) and Se-hyperdoped $\mathrm{Si}$ samples (gray). $\tau_{\text {fast }}$ (filled circles) and $\tau_{\text {slow }}$ (open circles) are the two decay time scales from in the model fit. The vertical line indicates the concentration at which the insulator-to-metal transition occurs. ${ }^{13}$ Inset shows normalized $1 /\left(N \tau_{\text {fast }}\right)$ for Sulfurhyperdoped silicon as a function of dopant concentration.

As controlled measurements (see supplementary material), ${ }^{23}$ we measure the carrier dynamics of two control samples: a bare Si substrate and an amorphous silicon sample that was amorphized by the implanted ions but was not treated with the ns-laser melting procedure. For the bare $\mathrm{Si}$ substrate after photoexcitation, the change in THz conductivity shows no appreciable decay within the 1-ns duration we probed, reflecting long carrier lifetimes as expected. For the amorphous silicon sample, the change in $\mathrm{THz}$ transmission is small (25 times smaller than the smallest time zero response shown in Figure 1) and quickly decays to zero within 2 ps, two orders of magnitude faster than the equivalent pulse-laser melted sample. Both controlled measurements agree with the reports in the literature. ${ }^{26,30}$

Since illuminating samples with light at $400 \mathrm{~nm}$ creates carriers from both the valence band and the IB to the conduction band, the excited carriers may decay through different pathways. Furthermore, the hyperdoped layer is thin enough that about $10 \%$ of the $400-n m$ pump photons are absorbed deeper than $200 \mathrm{~nm}$, at which point the dopant profile is no longer uniform and decreases toward zero on an approximately $100-n m$ length scale. ${ }^{21}$ To verify the origin of the short time carrier dynamics observed in the sample, we carried out pump-probe experiment with pump wavelength at $2300 \mathrm{~nm}$, which has energy less than the band gap energy of $\mathrm{Si}$, and the only excitation pathway is from the IB to the conduction band (illustrated in Figure 3). Figure 3 shows that the conductivity decay dynamics is identical for both pump wavelengths, suggesting the dynamics observed here is dominated by carriers relaxing from the conduction band to the intermediate states within the band gap.

We emphasize that in an intermediate band solar cell, the electrons in the conduction band must maintain a different quasi-Fermi level than that in the IB, so even if thermal reemission from trap states is possible, it cannot help IBPV performance. ${ }^{4}$ Thus, $\tau_{\text {fast }}$ is the relevant time scale for this work, as it characterizes the reestablishment of equilibrium between the conduction band and the IB carrier concentrations. There are a number of possible reasons why $\tau_{\text {slow }}$ is required to describe the photoconductivity decay. For example, the combination of carrier diffusion ${ }^{28,31}$ and inhomogeneity of $N$ as a function of depth ${ }^{11,21}$ introduces additional slow decay pathways. As electrons diffuse away from the hyperdoped region into regions with lower $N$ and higher carrier lifetime, the electrons sample a range of longer lifetimes which may give rise to the observed slow decay. This is supported by the fact that the relative weight $a_{2}$ is larger for longer $\tau_{\text {fast }}$ increasing the time for diffusion into the inhomogeneous region. Alternatively, a fraction of the carriers initially generated in the hyperdoped region could diffuse into the substrate, which has a long lifetime. $\tau_{\text {slow }}$ then characterizes the time for them to diffuse back into the hyperdoped region to recombine, with $a_{2}$ corresponding to the fraction of carriers that reach the substrate. Given the carrier mobility in these sample ranges from 40 to $1000 \mathrm{~cm}^{2} \mathrm{~V}^{-1} \mathrm{~s}^{-1},{ }^{23}$ the diffusion length in $\tau_{\text {fast }}$ increase from $10 \mathrm{~nm}$ for high $N$ to $700 \mathrm{~nm}$ for low $N$, comparable to the thickness of the hyperdoped layer for low $N$. For high $N$, more than $80 \%$ of the photoexcited carriers are trapped within 20 ps. In chalcogen-hyperdoped $\mathrm{Si}$, the threshold $N$ for the
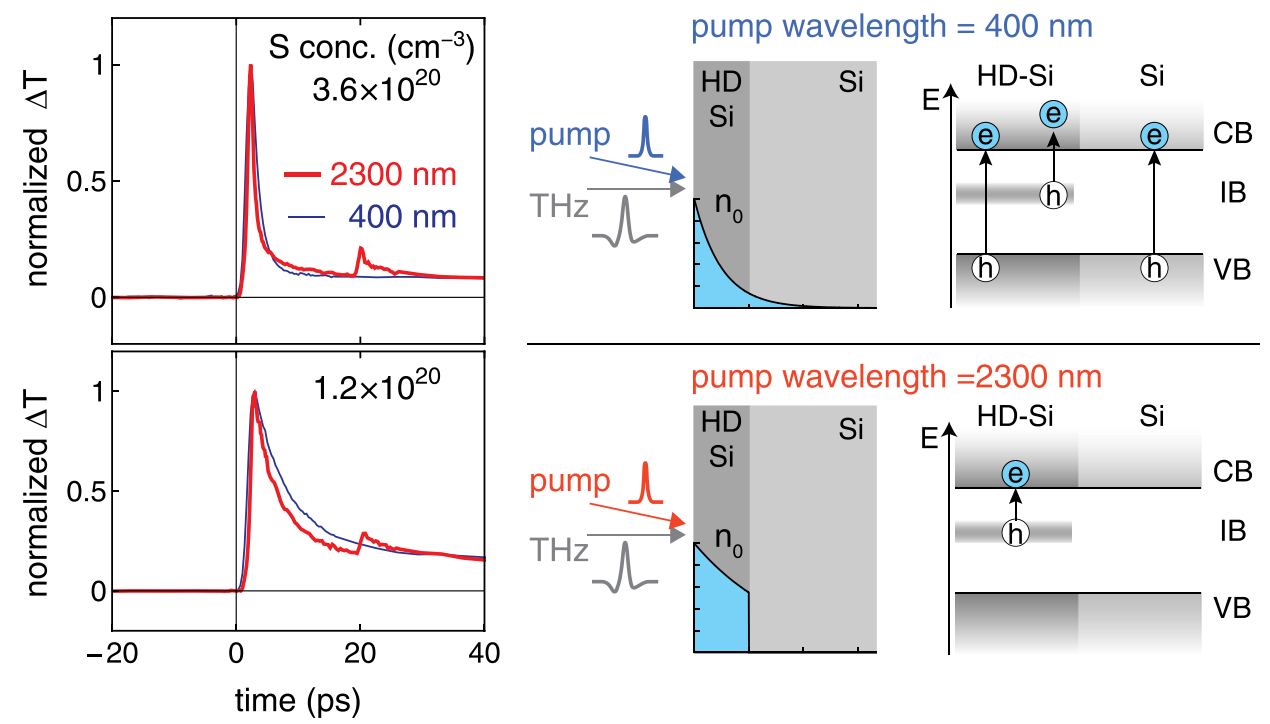

FIG. 3. Comparing changes of $\mathrm{THz}$ conductivity at different pump wavelengths at $400 \mathrm{~nm}$ (thin lines) and at $2300 \mathrm{~nm}$ (thick lines) and the data show the dynamics is independent of the pump wavelengths. For excitation at $2300 \mathrm{~nm}$, the pump beam is not completely absorbed, and the second pulse at 20 ps is due to reabsorption of the back-reflected light. Schematic on the right shows pump-probe experiment and the excitation pathways in HD-Si samples. The thickness of HD$\mathrm{Si}(200 \mathrm{~nm})$ and $\mathrm{Si}(800 \mu \mathrm{m})$ is not drawn to scale. 
IMT is determined by measuring temperature-dependent conductivity and the transition occurs around $3 \times 10^{20} \mathrm{~cm}^{-3}$ both S dopants and Se dopants. ${ }^{13}$ Figure 2 shows that the $\tau_{\text {fast }}$ continues to decrease for samples with concentrations above the transition threshold. Additional information can be obtained by comparing $\tau_{\text {fast }}$ to the electron trapping time $\tau_{e 0}$ in Shockley-Read-Hall recombination. The rate at which an electron in the conduction band is trapped by a set of empty trap states can be described by $1 / \tau_{e 0}=N_{T} \nu_{t h} \sigma_{e}(1-f)$, the product of the density of the traps $N_{T}$, the thermal velocity of electrons $\nu_{t h}$, the electron capture cross section $\sigma_{e}$, and the probability that a trap state is empty $(1-f)$, where $f$ is the filling fraction. ${ }^{7}$ Inset of Figure 2 shows normalized $1 /\left(N \tau_{\text {fast }}\right)$ and suggests that the electron trapping rate is increasing faster than $N$. The electron trapping rate increases could either be due to an increase of $\sigma_{e}$, contradicting the lifetime recovery theory or from carrier compensation and reduction of filling fraction $f$; no evidence for lifetime recovery is observed.

Finally, for each of the S-hyperdoped Si samples, we evaluate the figure of merit, which characterizes the potential for the material to produce excess electrons despite the short lifetimes. ${ }^{10}$ The figure of merit captures the balance between the absorption and recombination processes in the IB material and is written as, $\nu \equiv \frac{E_{g}}{q} \alpha^{2} \mu \tau$ where $\alpha$ is the sub-band gap absorption coefficient, $\mu$ is mobility of the carriers, and $E_{g}$ is the band gap energy. ${ }^{32}$ For $\nu \gg 1$, charge carriers can easily transport across the device layer and hence a solar cell made from such material generates additional power from the sub-band gap photons. ${ }^{33}$ The figure of merit can be evaluated for both electrons and holes. Since the quasi-Fermi level splitting is required for IBPV, both electrons and holes need to have a high figure of merit to achieve enhanced efficiency compared to a single junction device. With the carrier lifetimes obtained in this work, the figure of merit for electrons can be directly calculated using the measured $\tau_{\text {fast }}$ for carrier lifetime with values for $\alpha$ and $\mu$ obtained from Refs. 12,16 , and 34 (see supplementary material). ${ }^{23}$

The calculated figure of merit is shown in Figure 4 and shows that there is an optimal $N$ for the highest figure of merit. For the sample with $\mathrm{S}$ concentration at $1.2 \times 10^{20} \mathrm{~cm}^{-3}$, the figure of merit from our result is consistent with previous

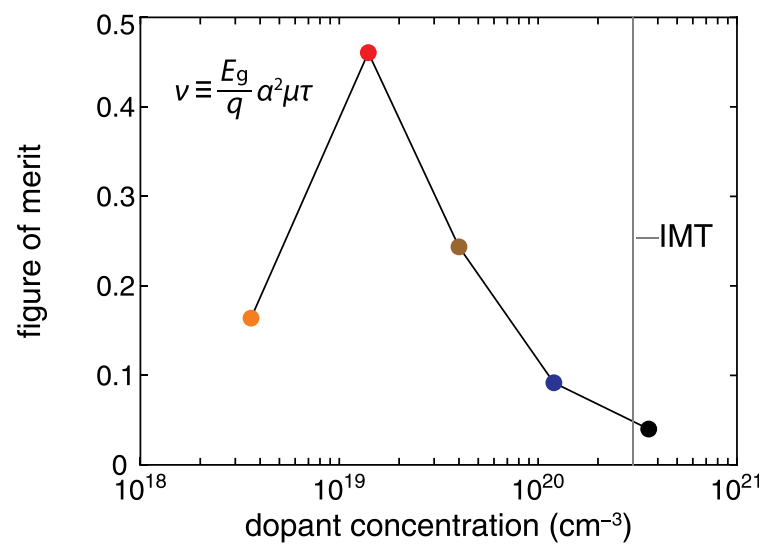

FIG. 4. Calculated figure of merit of S-hyperdoped Si samples. The sample with concentration above the IMT (vertical line) absorbs sub-band gap light most strongly but has the lowest figure of merit since both mobility and carrier lifetime are much lower. The sample with the highest figure of merit strikes a better balance between light absorption, mobility, and lifetime. reports. ${ }^{16}$ Our data show that at $N$ above the IMT, the sample has the lowest figure of merit (0.04). On the other hand, the highest figure of merit is just below 0.5 in a lower dose sample with $\mathrm{S}$ concentration at $1.4 \times 10^{19} \mathrm{~cm}^{-3}$.

By examining limiting cases, we show that the figure of merit as a function of $N$ would exhibit a maximum peak. Previous measurements show that $\alpha$ is proportional to $N .^{12}$ For low concentrations, $\mu$ is independent of $N$, and assuming constant $\sigma_{e}$ and $f$ at dilute concentrations, $\tau$ is proportional to $1 / N$. So at dilute concentrations, the figure of merit has a linear dependence on $N$ approaching zero at low concentrations. One the other hand, at high concentrations, we show that $\tau$ decreases faster than $1 / N$ (Figure 2 inset), and when carrier scattering is dominated by impurities rather than phonons, $\mu$ is proportional to $1 / N .^{35}$ In a highly doped regime, the figure of merit then decreases with increasing $N$. Consistent with our observations, the limiting cases suggest that there is an optimal dopant concentration at which the figure of merit for electron is maximized. In chalcogen-hyperdoped $\mathrm{Si}$, the most promising IBPV material is a sample with $N$ significantly below the IMT.

In conclusion, we present optical-pump/THz-probe measurements of carrier dynamics in chalcogen-hyperdoped $\mathrm{Si}$. The recombination dynamics is described by two exponential decay time scales. The fast decay time scale, corresponding to trapping an electron from the conduction band to the IB, ranges between 1 and $200 \mathrm{ps,} \mathrm{and} \mathrm{the} \mathrm{slow} \mathrm{decay}$ component is on the order of $1 \mathrm{~ns}$. No evidence for lifetime recovery is observed, and carrier lifetime continues to decrease with increasing $N$. The picosecond lifetime measured using this technique enables direct evaluation of materials potential for IBPV devices. We show that without lifetime recovery, the highest figure of merit in chalcogenhyperdoped Si occurs at $N$ significantly below the IMT.

We thank Sin-Cheng Siah and Professor Jay Mathews for valuable discussions. Research at Stanford was supported by the U.S. Department of Energy (DOE), Basic Energy Science, Materials Science and Engineering Division. The work at University of Ottawa was supported by NSERC. The work at Harvard was supported by the U.S. Army Research Office under Contract No. W911NF-12-1-0196. Research at MIT was supported by in part by the MIT-KFUPM Center for Clean Water and Energy; the National Science Foundation (NSF) grant under Contract No. ECCS-1102050; and the NSF and the DOE under NSF Contract No. EEC-1041895. This work was performed in part at the Center for Nanoscale Systems, a member of the National Nanotechnology Infrastructure Network, which was supported by the NSF under Award No. ECS-0335765.

${ }^{1}$ J. P. Mailoa, A. J. Akey, C. B. Simmons, D. Hutchinson, J. Mathews, J. T. Sullivan, D. Recht, M. T. Winkler, J. S. Williams, J. M. Warrender, P. D. Persans, M. J. Aziz, and T. Buonassisi, Nat. Commun. 5, 3011 (2014).

${ }^{2}$ M.-J. Sher, M. T. Winkler, and E. Mazur, MRS Bull. 36, 439 (2011).

${ }^{3}$ J. E. Carey, C. H. Crouch, M. Y. Shen, and E. Mazur, Opt. Lett. 30, 1773 (2005).

${ }^{4}$ A. Luque and A. Marti, Phys. Rev. Lett. 78, 5014 (1997).

${ }^{5}$ W. M. Wang, A. S. Lin, and J. D. Phillips, Appl. Phys. Lett. 95, 3 (2009); N. Lopez, L. A. Reichertz, K. M. Yu, K. Campman, and W. Walukiewicz, Phys. Rev. Lett. 106, 28701 (2011); S. Silvestre, A. Boronat, M. Colina, L. Castañer, J. Olea, D. Pastor, A. del Prado, I. Mártil, G. González-Díaz, A. 
Luque, E. Antolín, E. Hernández, I. N. Ramiro, I. Artacho, E. López, and A. Martí, Jpn. J. Appl. Phys., Part 1 52, 122302 (2013); T. Tanaka, M. Miyabara, Y. Nagao, K. Saito, Q. Guo, M. Nishio, K. M. Yu, and W. Walukiewicz, Appl. Phys. Lett. 102, 052111 (2013); T. Sogabe, Y. Shoji, M. Ohba, K. Yoshida, R. Tamaki, H.-F. Hong, C.-H. Wu, C.-T. Kuo, S. Tomić, and Y. Okada, Sci. Rep. 4, 4792 (2014).

${ }^{6}$ A. Luque, A. Marti, E. Antolin, and C. Tablero, Physica B: Condens. Matter 382, 320 (2006).

${ }^{7}$ W. Shockley and W. Read, Phys. Rev. 87, 835 (1952).

${ }^{8}$ E. Antolin, A. Marti, J. Olea, D. Pastor, G. Gonzalez-Diaz, I. Martil, A. Luque, E. Antolín, A. Martí, G. González-Díaz, and I. Mártil, Appl. Phys. Lett. 94, 42115 (2009).

${ }^{9}$ A. Karoui, Appl. Phys. Lett. 101, 236101 (2012).

${ }^{10}$ J. J. Krich, B. I. Halperin, and A. Aspuru-Guzik, J. Appl. Phys. 112, 13707 (2012).

${ }^{11}$ S. H. Pan, D. Recht, S. Charnvanichborikarn, J. S. Williams, and M. J. Aziz, Appl. Phys. Lett. 98, 121913 (2011).

${ }^{12}$ I. Umezu, J. M. Warrender, S. Charnvanichborikarn, A. Kohno, J. S. Williams, M. Tabbal, D. G. Papazoglou, X.-C. Zhang, and M. J. Aziz, J. Appl. Phys. 113, 213501 (2013).

${ }^{13}$ E. Ertekin, M. T. Winkler, D. Recht, A. J. Said, M. J. Aziz, T. Buonassisi, and J. C. Grossman, Phys. Rev. Lett. 108, 26401 (2012); M. T. Winkler, D. Recht, M.-J. Sher, A. J. Said, E. Mazur, and M. J. Aziz, ibid. 106, 178701 (2011).

${ }^{14}$ D. Recht, D. Hutchinson, T. Cruson, A. DiFranzo, A. McAllister, A. J. Said, J. M. Warrender, P. D. Persans, and M. J. Aziz, Appl. Phys. Express 5, 041301 (2012).

${ }^{15}$ P. D. Persans, N. E. Berry, D. Recht, D. Hutchinson, H. Peterson, J. Clark, S. Charnvanichborikarn, J. S. Williams, A. DiFranzo, M. J. Aziz, and J. M. Warrender, Appl. Phys. Lett. 101, 111105 (2012).

${ }^{16}$ J. T. Sullivan, C. B. Simmons, J. J. Krich, A. J. Akey, D. Recht, M. J. Aziz, and T. Buonassisi, J. Appl. Phys. 114, 103701 (2013).

${ }^{17}$ K. Reimann, Rep. Prog. Phys. 70, 1597 (2007).

${ }^{18}$ C. W. White, S. R. Wilson, B. R. Appleton, and F. W. Young, J. Appl. Phys. 51, 738 (1980).

${ }^{19}$ M. Tabbal, T. Kim, J. M. Warrender, M. J. Aziz, B. L. Cardozo, and R. S. Goldman, J. Vac. Sci. Technol. B 25, 1847 (2007).

${ }^{20}$ J. T. Sullivan, R. G. Wilks, M. T. Winkler, L. Weinhardt, D. Recht, A. J. Said, B. K. Newman, Y. Zhang, M. Blum, S. Krause, W. L. Yang, C.
Heske, M. J. Aziz, M. Bär, and T. Buonassisi, Appl. Phys. Lett. 99, $142102(2011)$.

${ }^{21}$ B. P. Bob, A. Kohno, S. Charnvanichborikarn, J. M. Warrender, I. Umezu, M. Tabbal, J. S. Williams, and M. J. Aziz, J. Appl. Phys. 107, 123506 (2010).

${ }^{22}$ H. Wen and A. Lindenberg, Phys. Rev. Lett. 103, 023902 (2009); H. Wen, D. Daranciang, and A. M. Lindenberg, Appl. Phys. Lett. 96, 161103 (2010); M. D. Thomson, M. Kreß, T. Löffler, and H. G. Roskos, Laser Photon. Rev. 1, 349 (2007); P. C. M. Planken, H.-K. Nienhuys, H. J. Bakker, and T. Wenckebach, J. Opt. Soc. Am. B 18, 313 (2001).

${ }^{23}$ See supplementary material at http://dx.doi.org/10.1063/1.4892357 for details in data analysis results and additional $\mathrm{THz}$ probe measurements on Se-hyperdoped Si and reference samples.

${ }^{24}$ F. Gao, G. Carr, C. Porter, D. Tanner, G. Williams, C. Hirschmugl, B. Dutta, X. Wu, and S. Etemad, Phys. Rev. B 54, 700 (1996).

${ }^{25}$ F. A. Hegmann and K. P. Lui, Proc. SPIE 4643, 31-41 (2002).

${ }^{26} \mathrm{G}$. Li, D. Li, Z. Jin, and G. Ma, Opt. Commun. 285, 4102 (2012).

${ }^{27}$ K. P. H. Lui and F. A. Hegmann, Appl. Phys. Lett. 78, 3478 (2001).

${ }^{28}$ M. C. Beard, G. M. Turner, and C. A. Schmuttenmaer, J. Appl. Phys. 90, 5915 (2001).

${ }^{29}$ M. C. Beard, G. M. Turner, and C. A. Schmuttenmaer, Nano Lett. 2, 983 (2002).

${ }^{30}$ K. P. H. Lui and F. A. Hegmann, J. Appl. Phys. 93, 9012 (2003).

${ }^{31}$ M. Beard, G. Turner, and C. Schmuttenmaer, Phys. Rev. B 62, 15764 (2000).

${ }^{32}$ Reference 10 has the built-in voltage $V_{b i}$ in place of $E_{g} / q$. Since $V_{b i}$ can approach $E_{g} / q$ and $E_{g}$ is a material property of the IB region alone, it is preferable to replace $V_{b i}$ with $E_{g} / q$.

${ }^{33}$ J. J. Krich, A. H. Trojnar, L. Feng, K. Hinzer, and A. W. Walker, Proc. of SPIE 8981, 89810 O (2014).

${ }^{34}$ C. B. Simmons, A. J. Akey, J. P. Mailoa, D. Recht, M. J. Aziz, and T. Buonassisi, Adv. Funct. Mater. 24, 2852 (2014); M. Winkler, "Non-equilibrium chalcogen concentrations in silicon: Physical structure, electronic transport, and photovoltaic potential," Ph.D. dissertation (Harvard University, 2009); C. B. Simmons, A. J. Akey, J. J. Krich, J. T. Sullivan, D. Recht, M. J. Aziz, and T. Buonassisi, J. Appl. Phys. 114, 243514 (2013).

${ }^{35}$ S. M. Sze, Physics of Semiconductor Devices (Wiley-Interscience, New York, 1981), p. 28. 